\title{
Archéopages
}

Archéopages

Archéologie et société

\section{Construction en terre crue au Néolithique moyen I}

Le tertre du monument 29 de Fleury-sur-Orne

Building with earth in the Middle Neolithic I. The mound of Monument 29 at Fleury-sur Orne (Calvados)

Construcción de barro en el Neolítico Medio I. El túmulo del monumento 29 de Fleury-sur-Orne (Calvados)

\section{Emmanuel Ghesquière, David Giazzon et Julia Wattez}

\section{CpenEdition}

\section{Journals}

Édition électronique

URL : https://journals.openedition.org/archeopages/1146

DOI : 10.4000/archeopages. 1146

ISSN : 2269-9872

Éditeur

INRAP - Institut national de recherches archéologiques préventives

Édition imprimée

Date de publication : 1 mars 2016

ISSN : 1622-8545

\section{Référence électronique}

Emmanuel Ghesquière, David Giazzon et Julia Wattez, « Construction en terre crue au Néolithique moyen I », Archéopages [En ligne], 42 | 04-07/2015, mis en ligne le 11 octobre 2017, consulté le 07 juin 2021. URL : http://journals.openedition.org/archeopages/1146 ; DOI : https://doi.org/10.4000/ archeopages. 1146 


\section{Construction en terre crue}

\section{au Néolithique moyen I \\ Le tertre du monument 29 de Fleury-sur-Orne}

Emmanuel Ghesquière Irrap, UMR 656, “CReAAH"

David Giazzon ${ }^{\text {Irrap }}$

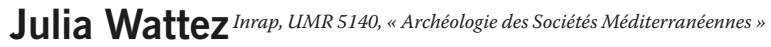

\section{8}

1. Diagnostic réalisé en 2012 sous la direction de David Flotté, Inrap ; fouille des « Hauts de l'Orne» réalisée de mai à décembre 2014 sous la direction d'Emmanuel Ghesquière, Inrap.
La nécropole de Fleury a été découverte par photographie aérienne aux débuts des années 1990. Plusieurs diagnostics et sondages ont alors été réalisés, mais l'importance du site a pendant 20 ans repoussé l'aménagement d'une zone pourtant située dans un environnement périurbain dense. Ce n'est qu'en 2012 qu'un projet ambitieux de l'agglomération a permis de faire réaliser un diagnostic complet (Flotté, 2013), suivi par une fouille en $2014^{1}$. Cette dernière a concerné essentiellement les vestiges funéraires néolithiques et, de façon anecdotique, ceux de la seconde guerre mondiale. La nécropole néolithique est installée dans un large vallon plan (moins de $1 \%$ de pente), avec actuellement un faible recouvrement terreux $(0,25 \mathrm{~m})$ qui surmonte la plaquette calcaire désagrégée par les alternances climatiques de la dernière glaciation. On note la proximité de la vallée de l'Orne (500 m) et du littoral $(15 \mathrm{~km})$. La fouille a couvert une surface triangulaire de 21 hectares d'un seul tenant [ill. 1]. Elle a mis en évidence 26 monuments de type Passy, deux cairns (dont un était connu et déjà fouillé) et trois alignements destinés à installer des menhirs ou des mégaxyles. Les monuments de type Passy mesurent, à Fleury-sur-Orne, entre 12 et $372 \mathrm{~m}$ de longueur, respectant une direction générale estouest et suivant un tracé légèrement sinueux. Leur profil est parfois étroit, sous forme de tranchée (palissadée ?), pour les monuments en « épingle à cheveux », ou ouvert et large pour les monuments trapézoïdaux. Les seuls vestiges retrouvés dans les fossés sont quelques outils en os (omoplates de bovidés en particulier) et des pics massifs en grès rouge de provenance locale, utilisés dans le cadre du creusement des fossés. Ces monuments contiennent en règle générale une unique sépulture, pas toujours retrouvée lors de la fouille (tombe hors sol ?), rarement deux. Les plus caractéristiques du site abritent un archer, les plus riches sont accompagnés par des offrandes de moutons. L'un des monuments du type Passy (monument 29), dont le tertre a été préservé par l'installation d'un chemin antique qui le recouvrait complètement, a particulièrement attiré l'attention. Le présent article s'attache à comprendre son mode de construction et son évolution chronologique.

\section{La physionomie du monument 29}

Long de $146 \mathrm{~m}$, le monument 29, orienté estouest, est le plus large du site : 14,6 m à l'extrémité occidentale et $57,5 \mathrm{~m}$ à l'extrémité orientale (fossés compris). La partie inscrite entre les deux fossés mesure entre $9 \mathrm{~m}$ à l'ouest et 26,5 $\mathrm{m}$ à l'est [ill. 2]. Ces deux fossés présentent une physionomie comparable, avec un creusement subvertical vers l'intérieur du monument et en pente douce vers l'extérieur, d'une profondeur allant de quelques décimètres à l'ouest à $1,5 \mathrm{~m}$ à l'est. Leurs dimensions (de l'ordre de $1000 \mathrm{~m}^{3}$ extraits) sont sans commune mesure avec les autres fossés du site. Deux datations ${ }^{14} \mathrm{C}$ ont été réalisées sur les ossements ; les deux dates, bien homogènes, 5730 $\pm 30 \mathrm{BP}$ et $5770 \pm 30 \mathrm{BP}$, suggèrent une fourchette combinée entre 4685 et $4540 \mathrm{O}$ cal BC pour la construction du monument.

Le tertre, qui présente une forme trapézoïdale très allongée, avec un doublement de la largeur entre les deux extrémités, est long de 80 m environ (extrémité ouest très dégradée), en prenant en compte l'extension parementée de $3,5 \mathrm{~m}$ de l'extrémité est. Il est large de $11 \mathrm{~m}$ (estimé) à l'ouest et de $23 \mathrm{~m}$ à l'est pour la terminaison en mottes de 


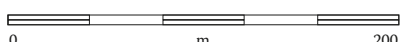

Structures en creux de la nécropole Structures en élévation, tertre du monument 29 ou cairns des monuments 9 et 40

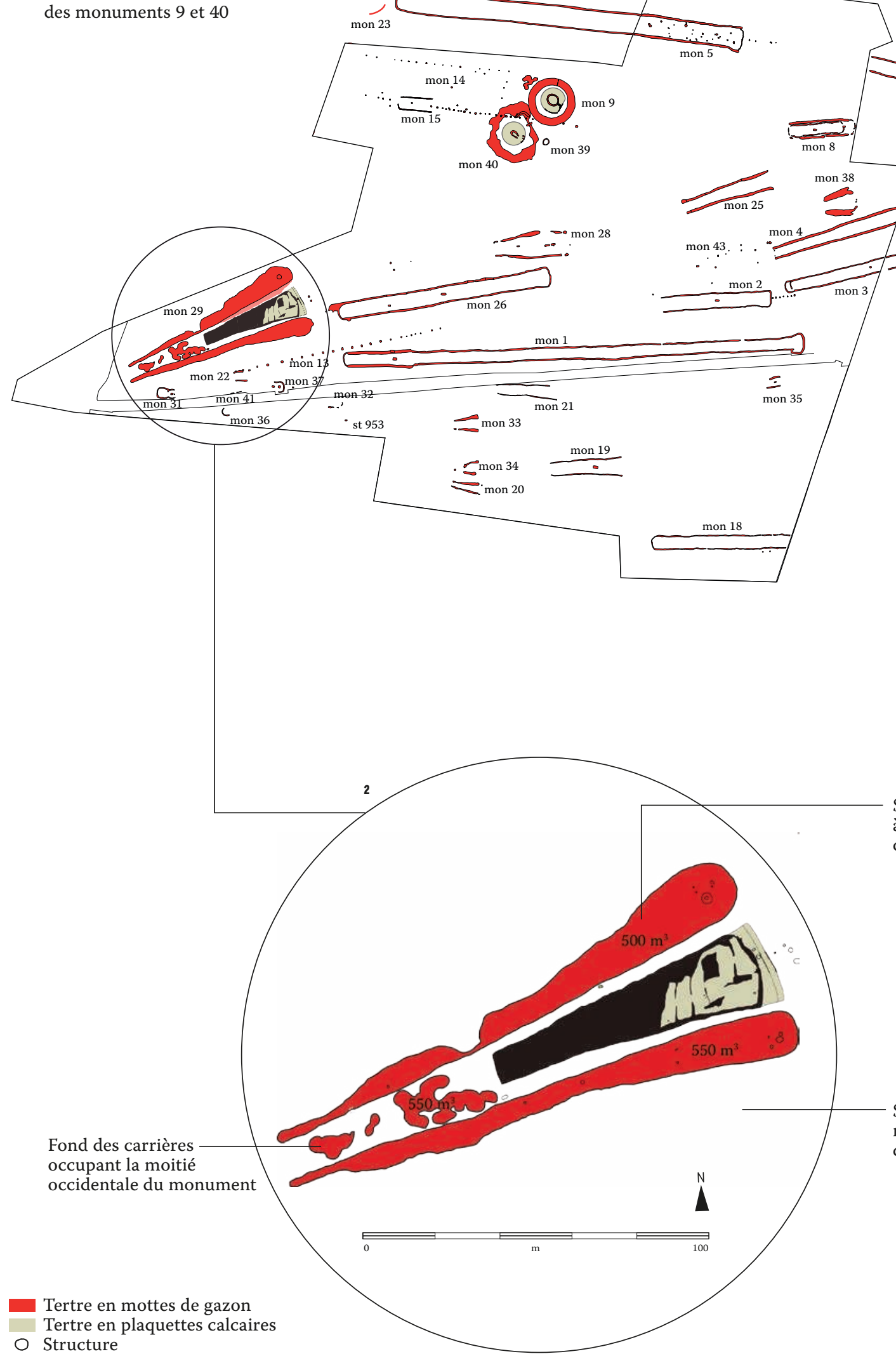

1. Plan de la nécropole de Fleury-sur-Orne avec en encadré la zone fouillée en 2014.

2. Plan simplifié du

monument 29 en haut. avec ses deux puissants fossés, ses carrières occupant l'espace entre les fossés à l'ouest et le tertre fossilisé sous un chemin romain à l'est. En bas, coupe simplifiée du monument : en noir, le tertre, en bleu, les couches d'effondrement néolithiques dans les fossés et en rouge, les couches de remplissage post-romaines.

Structures nécessaires

à la récupération des plaquettes calcaires du tertre $\left(1600 \mathrm{~m}^{3}\right)$

Surface de tapis de gazon nécessaires à l'élévation du tertre $\left(900 \mathrm{~m}^{3}\right)$ 

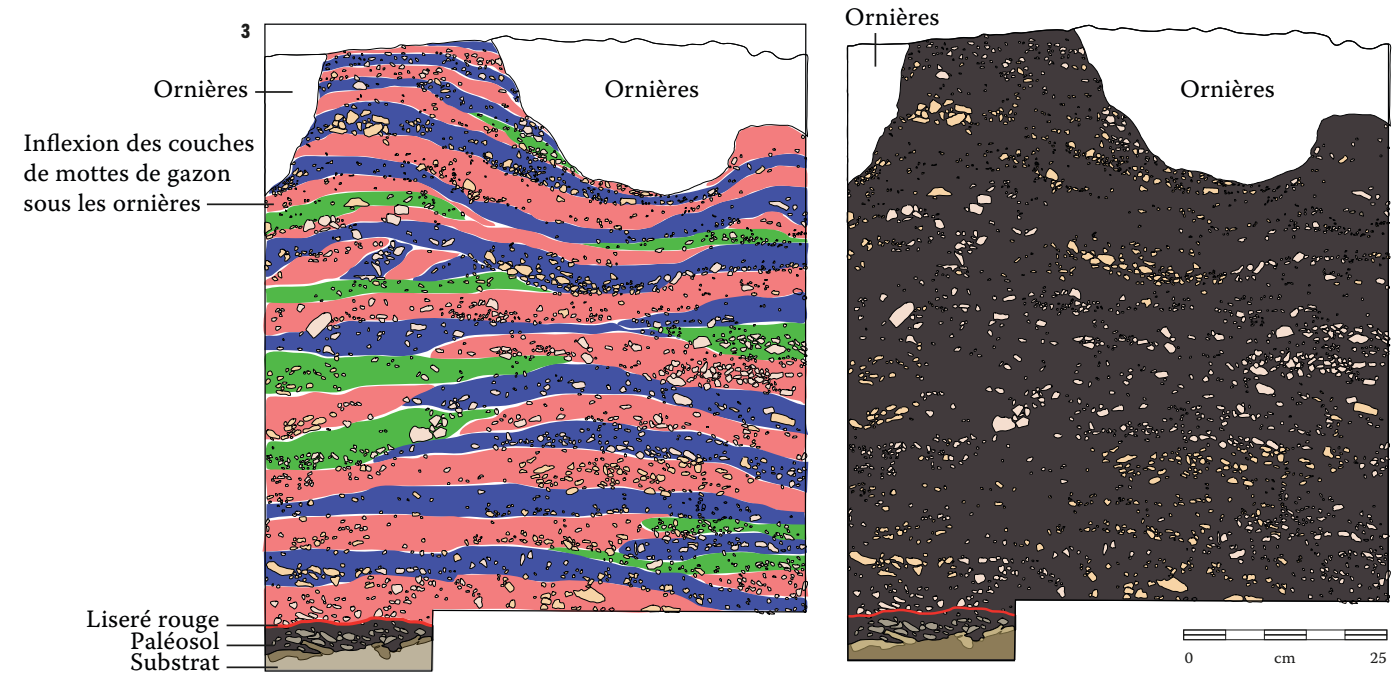

3. En haut, le dessin

d'après photo d'un détail

d'une coupe du tertre

permet d'individualiser

les couches de tapis de

gazon empilées d'après

les gravillons calcaires

entraînés par les

systèmes racinaires; en

bas, la fouille à plat du

tertre permet également

d'individualiser les

différents tapis de gazon

empilés et croisés.

$1^{\mathrm{er}}$ niveau

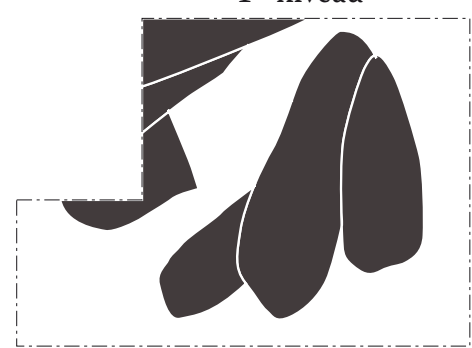

$4^{\mathrm{e}}$ niveau

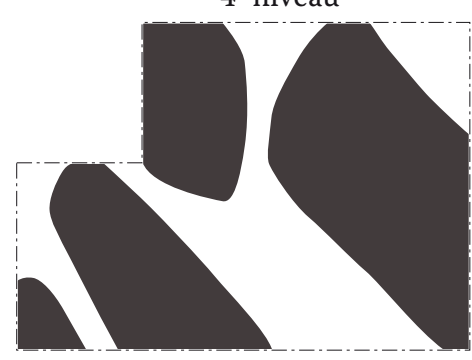

$2^{\mathrm{e}}$ niveau

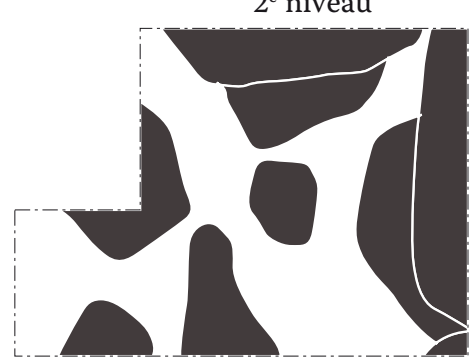

$5^{\mathrm{e}}$ niveau

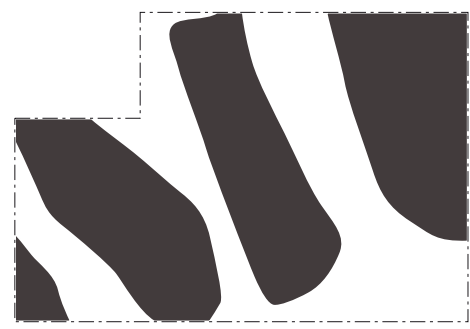

$3^{\mathrm{e}}$ niveau

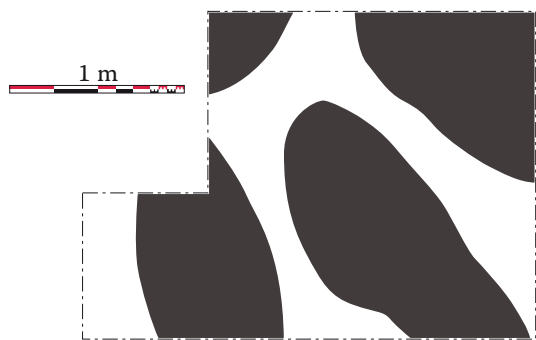

$6^{\mathrm{e}}$ niveau

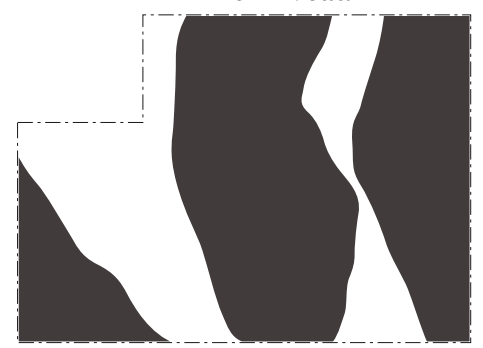

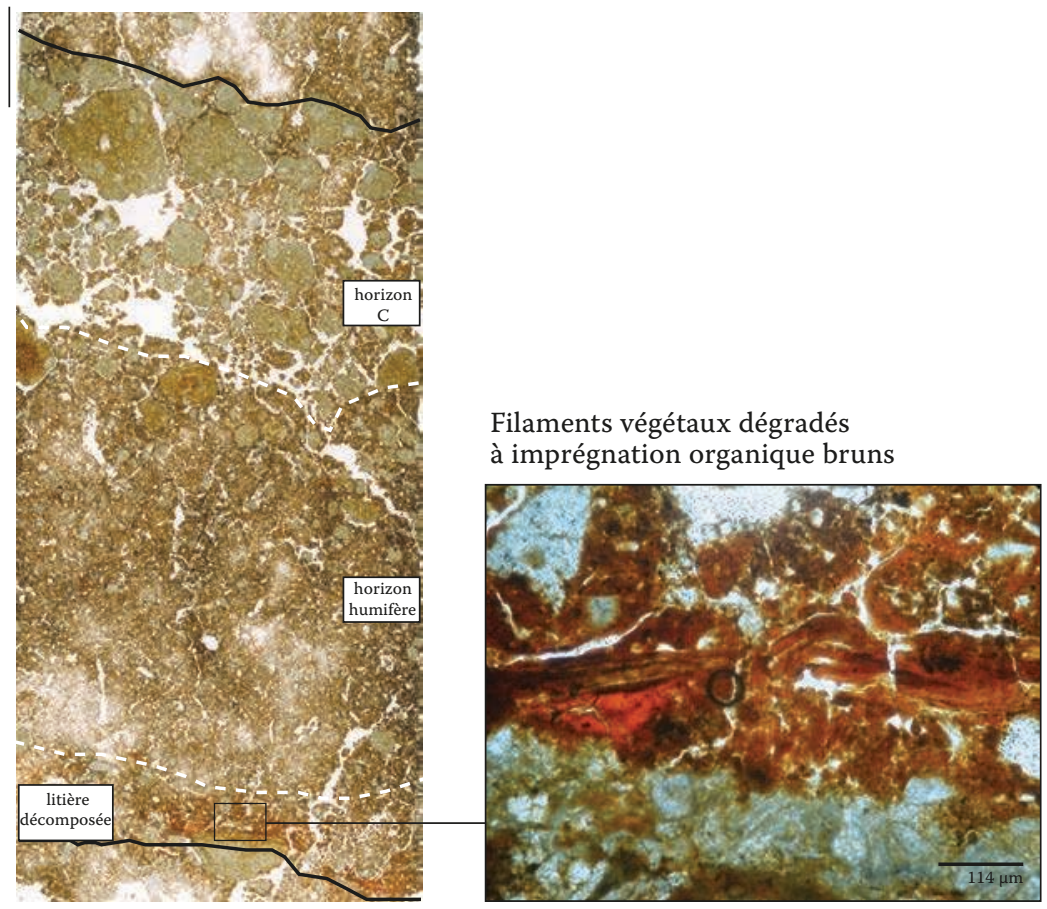

4. Microstratigraphie d'une motte de gazon prélevée dans un rendosol (rendzine, sol humifère développé sur substrat calcaire) : elle présente un profil pédologique inverse avec l'horizon d'altération en haut et le reste de couvert vegetal

en bas. La structure d'origine du sol est conservée, légèrement déstructurée par les opérations de mise en œuvre (prélèvement, dépôt et légère compaction), ce qui atteste de la très bonne cohésion de l'horizon humifère liée au

complexe organo-minéral et au réseau racinaire, les graviers pouvant jouer le rôle d'armature. 
gazon. La terminaison parementée, encore plus large, est difficilement estimable en raison de l'absence des angles ; elle serait de l'ordre de $25 \mathrm{~m}$. Le tertre est conservé sur une hauteur de o,8 m, le maximum de conservation correspondant au parement en mottes de gazon de la façade orientale. La répartition des éboulis de l'extrémité orientale en pierre (pour partie dans le fossé sud) témoignerait d'une hauteur d'origine de 2,5 à $3 \mathrm{~m}$ à l'est. Le tertre est établi sur un rendosol humifère (horizon ACA de rendzine de 3 à $7 \mathrm{~cm}$ d'épaisseur) qui se développe sur des formations calcaires (Bathonien) [ill. 3 et 4]. D'autres éléments laissent penser que les autres monuments du site présentaient le même mode de mise en œuvre.

\section{Les matériaux de construction}

Trois matériaux de construction ont été utilisés dans l'élaboration du tertre, parfois associés entre eux : des mottes de gazon, des plaquettes calcaires et des limons.

Les mottes de gazon sont un des matériaux privilégiés : ils consistent en tapis (ou blocs ?) découpés dans le rendosol qui ont été empilés sur toute la partie ouest du tertre [ill. 3]. Ils forment des couches régulières, continues ou lenticulaires, formées par l'alternance de dépôts limono-sableux brun noir (horizon humifère avec son réseau racinaire), de 3 à $6 \mathrm{~cm}$ d'épaisseur, et de dépôts de gravillons et de graviers calcaires de 1 à $4 . \mathrm{cm}$ d'épaisseur (horizon $C$, horizon d'altération du substrat calcaire) [ill. 3 et 4]. L'étude micromorphologique confirme ces observations, avec la mise en évidence en lame mince d'une motte de gazon inversée, ayant conservé en partie les restes très dégradés de la base du couvert végétal (litière décomposée) [ill. 4]. La faible épaisseur des «tapis de gazon » renvoie à celle des horizons humifères de rendosol, souvent peu profonds, et témoigne d'un environnement de prélèvement identique à celui observé sous le tertre. Il est donc probable que le prélèvement ait été réalisé autour du monument.

Les plaquettes calcaires correspondent sans doute au matériau le plus utilisé si l'on en juge par l'extension et le volume des fossés/carrières encadrant le tertre. Ce calcaire, exploité dans les 1,5 $\mathrm{m}$ maximum de profondeur des fossés, est de qualité moyenne, fragmenté par les cryoturbations glaciaires, et se présente en plaquettes de o,1 à $0,3 \mathrm{~m}$ de longueur, rarement plus.

Les limons ont fait l'objet d'une utilisation extrêmement restreinte, participant au remplissage de quelques caissons et de refends internes. Leur provenance est peut-être un peu plus éloignée du tertre que les calcaires et les tapis de gazon, mais ne dépasse pas quelques centaines de mètres, vers le sud ou l'ouest.

L'association entre les plaquettes calcaires et les mottes de gazon parfois observée pourrait avoir contribué à armer les tapis de gazon et assurer la stabilité du tertre [ill. 5]. Toutefois, on observe plutôt une exclusion de ces deux matériaux très différents, chacun s'appropriant l'est ou l'ouest de l'ouvrage, bien que les tapis de gazon continuaient vers l'est à tenir le rôle de parement et de refends internes.

\section{Le mode de construction}

Les limites extérieures du monument de Fleury sont constituées d'un parement réalisé en mottes/ tapis de gazon. Ces tapis sont empilés de manière à faire adopter un fruit au parement de l'ordre de $60^{\circ}$ vers l'intérieur du monument, destiné à éviter un effritement trop rapide, d'autant plus que la végétation devait reprendre en surface. $\mathrm{Ce}$ parement en mottes de gazon est surtout visible sur le côté sud du tertre où il est conservé sur o,6 m de hauteur. La façade orientale se présente dans un premier temps comme un parement en mottes/ tapis de gazon convexe, qui devait atteindre une hauteur estimée à $2 \mathrm{~m}$. Le côté nord, nettement plus érodé et détruit par des carrières romaines, n'est plus présent que sous forme de lambeaux.

La fouille du tertre a permis d'observer la méthode utilisée pour l'élaboration interne du tertre. Les zones élevées en plaquettes calcaires ont livré les témoins de banquettes de refend de tapis de gazon utilisées pour marquer au sol l'emprise du monument, installés directement sur le paléosol.

Les murets en tapis de gazon déterminent donc : la paroi externe du monument (de 0,8 à $1 \mathrm{~m}$ de largeur), les murets de refend (de 0,3 à o,4 $\mathrm{m}$ de largeur) et un quadrillage orthogonal ou curviligne (de peu d'épaisseur) qui prend appui sur les murets de refend [ill. 6]. Le parement extérieur présente un état de conservation optimum sur sa façade orientale et sur une partie de son côté sud. Il permet d'observer son fruit vers l'intérieur pour assurer sa stabilité et son épaisseur constante (sur les o,8 m de hauteur maximale conservée). Les refends internes pour leur part sont très certainement partiels. Leur première caractéristique est qu'ils sont plus épais près des parois extérieures qu'au centre du monument. La seconde caractéristique est qu'ils sont plus épais à leur base qu'à $0,5 \mathrm{~m}$ de hauteur (maximum conservé). Il y a une forte probabilité pour qu'ils ne soient pas présents sur toute la hauteur du monument, comme le suggère leur rétrécissement rapide en hauteur. Il est toutefois difficile d'en juger du fait d'une conservation incomplète du monument. Les derniers murets en mottes de gazon qui délimitent des espaces carrés ou arrondis entre les refends présentent une hauteur variable : au moins $0,4 \mathrm{~m}$ dans la partie ouest du monument, où ils sont peu fréquents, mais seulement $0,1 \mathrm{~m}$ dans toute la moitié orientale où ils ne semblent constituer que des marques au sol inutiles lorsque le monument commence à s'élever à quelques décimètres du sol. Contrairement aux murets de refend, ils ne semblent pas avoir un rôle dans la cohésion de la structure mais servent plutôt de repères au début de la construction. 


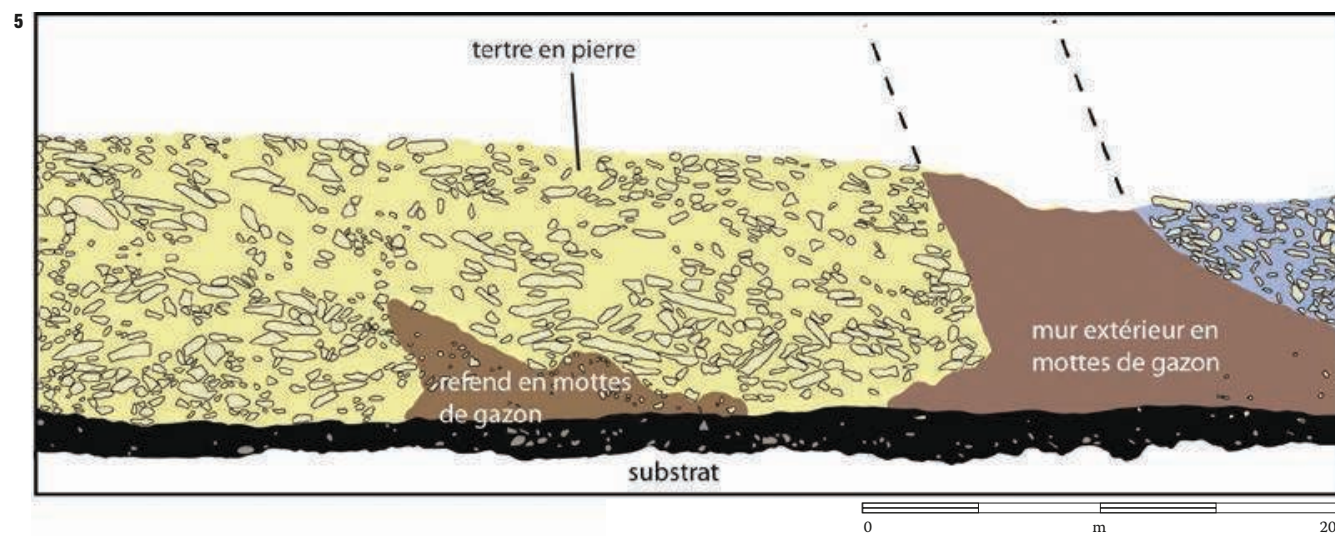

5. En haut, le parement externe sud du tertre

constitué d'un mur en

tapis de gazon limitant

une masse en matériaux

calcaires ; en bas, un

muret de refend interne

en tapis de gazon

délimite le remplissage

de deux caissons, l'un

majoritairement

composé de tapis de

gazon empilés, l'autre

dominé par l'empilement

de matériaux calcaires,

plaquettes et débris,

provenant probablement

du creusement des

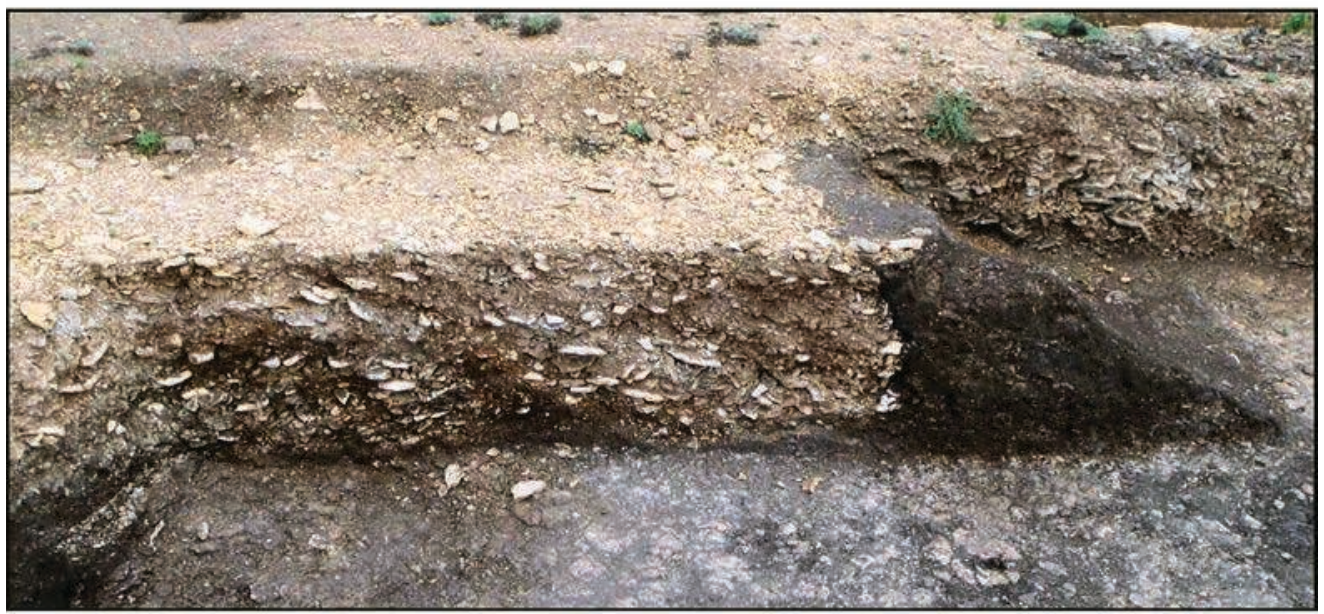

fossés latéraux

Le remplissage des caissons délimités au sol par des murets plus ou moins élevés en mottes de gazon a permis de comprendre la façon dont la construction a été menée. Les deux tiers occidentaux sont construits en tapis de gazon. Quelques éléments internes comme une possible cloison axiale (largement perturbée par une canalisation d'eau moderne) et des cloisons curvilignes (en limons et éléments calcaires) témoigneraient éventuellement de marqueurs au sol. Les tapis de gazon sont empilés dans ces caissons de manière à partiellement se croiser et se recouvrir, pour assurer une stabilité à l'ensemble et/ou un niveau relativement plan.

Le tiers oriental du monument est construit uniquement en plaquettes calcaires déposées à plat dans les espaces entre les murets de refends en mottes de gazon. Leur disposition présente un soin particulier, sans doute pour assurer la stabilité de l'ensemble. Dans l'état de leur découverte (niveaux inférieurs du tertre), ces couches calcaires sont homogènes et lacunaires.

Entre la partie du tertre construite en mottes de gazon et celle construite en pierre, la distinction très nette au niveau du décapage laissait présager un possible agrandissement du tertre. Or, la fouille de cette partie et le relevé du remplissage d'une coupe pratiquée dans ce secteur ont livré une image tout à fait incompatible avec une extension. En effet, le passage d'un mode de remplissage à l'autre s'opère de part et d'autre d'un muret de refend monté en mottes de gazon. Le remplissage de chaque côté est constitué de couches disposées en biais, partie haute contre le muret, qui témoignent du rétrécissement du muret de sa base vers son sommet (observable par ailleurs sur tous les murets de refend). Ces couches de part et d'autre du muret ne montrent pas comme en surface l'homogénéité à laquelle nous aurions pu nous attendre (mottes de gazon à l'ouest et pierres à l'est), mais une succession de couches pierreuses et de tapis de gazon, progressivement plus caillouteuses vers l'est. Ce passage progressif du gazon à la pierre, de part et d'autre d'un muret de refend, témoigne bien d'une progression significative du remplissage d'un monument unique sur toute sa longueur, si l'on excepte la réfection du parement de façade oriental.

\section{Les volumes de matériaux mis en œuvre}

Les volumes de pierre extraits dans les fosséscarrières et dans les carrières internes du monument ont pu être appréciés sous forme d'une approximation. Différents facteurs comme les reprises de carrière à la période antique dans le fossé nord, l'arasement de toute la partie interne du monument dans sa moitié ouest ou encore l'arasement du substrat sur les extérieurs du fossé ne permettent pas un calcul précis mais plutôt $a$ minima. En volume creusé préservé, le prisme allongé du fossé nord est de l'ordre de $500 \mathrm{~m}^{3}$. Celui du fossé sud, un peu plus profond, est de l'ordre de
$550 \mathrm{~m}^{3}$. Les carrières internes, qui ont entamé le substrat entre les fossés d'au moins o,5 m sur les $60 \mathrm{~m}$ de sa partie occidentale (un peu plus profondément par endroits également), représentent un volume approximatif de $550 \mathrm{~m}^{3}$ également. Le total extrait est donc de l'ordre de $1600 \mathrm{~m}^{3}$. Avec ce volume de plaquettes calcaires, on peut considérer comme raisonnable leur emploi pour élever le tertre, même si cela reste une hypothèse basée en premier lieu sur l'identité du matériau et sa proximité. Dans l'état actuel du tertre (avant la fouille), seuls les $30 \mathrm{~m}$ orientaux du tertre sont construits en calcaire, le reste du tertre est exclusivement constitué d'un empilement de tapis de gazon. À l'origine, la répartition du matériau pierreux pouvait être beaucoup plus large et reposer sur des couches même épaisses de tapis de gazon. Sans exclure cette hypothèse, il convient de calculer la possibilité d'élévation si le matériau pierreux extrait avait été utilisé uniquement pour l'élévation du tiers oriental du tertre. Un calcul rapide volume permet de constater que l'extrémité de $30 \mathrm{~m}$ de longueur sur $20 \mathrm{~m}$ de largeur devrait s'élever à 2,6 $\mathrm{m}$ pour utiliser toute la pierre extraite, éventuellement un peu plus à l'est si une pente est respectée. En l'état, ce chiffre est tout à fait dans les normes de ce que l'on peut attendre d'un tel monument (par comparaison avec Colombiers-sur-Seulles [Calvados] par exemple).

Si l'on retient cette hypothèse d'un monument plus haut à l'est qu'à l'ouest, comme semble également le suggérer l'état de conservation général du tertre, on peut calculer le cubage de mottes de gazon nécessaire à son élévation. Toute la surface arrière $(50 \mathrm{~m})$ sur une largeur de $15 \mathrm{~m}$ en moyenne aurait pu, selon cette hypothèse de travail, présenter une pente entre $2 \mathrm{~m}$ à l'est et $\mathrm{o}, 10 \mathrm{~m}$ à l'ouest, soit un volume de $750 \mathrm{~m}^{3}$ pour le tertre et 150 de plus pour le parement et les refends internes de la partie pierreuse. Par ailleurs, l'observation de l'alternance des tapis de gazon nous apprend que la hauteur moyenne du tapis de gazon utilisé à Fleury est de l'ordre de $5 \mathrm{~cm}$, ce qui correspond également à la hauteur moyenne du paléosol sous le tertre. Cette faible épaisseur se traduit donc par l'empilement nécessaire de 20 couches de gazon pour obtenir une hauteur d'un mètre (moyenne de hauteur estimée). Les $900 \mathrm{~m}^{3}$ nécessaires à la construction du tertre correspondraient donc à un décapage du substrat de l'ordre de 18 ooo $\mathrm{m}^{2}$ (1,8 ha). L'extraction du calcaire aurait ainsi contribué, si elle a bien été réalisée autour du monument, à en réhausser la visibilité. L'utilisation des limons dans l'élaboration du tertre est impossible à estimer dans l'état actuel de préservation du monument, seuls quelques mètres cubes ayant pu être observés dans une petite zone. Ce matériau a bien sûr pu être plus largement utilisé dans les couches supérieures arasées du tertre, mais aucun élément ne permet d'en juger. Sa provenance un peu plus éloignée (400 $\mathrm{m}$ ?) pourrait expliquer son utilisation négligeable par rapport aux deux autres matériaux. 


\section{Les deux états du monument}

L'observation de l'extrémité orientale du tertre nous montre un parement convexe en mottes de gazon, préservé sur o,8 $\mathrm{m}$ de hauteur, qui présente un renflement vers l'extérieur, correspondant probablement à la poussée des matériaux. Cette façade convexe comporte par ailleurs en son milieu et à son aplomb une structure circulaire profonde interprétée comme le support d'un mégaxyle dont la hauteur devait dépasser celle du monument [ill. 2]. Ces deux éléments (renflement et mégaxyle) suggèrent qu'il s'agit de la façade d'origine du tertre. Son obsolescence résulte probablement de la hauteur du monument à l'est et de l'inévitable poussée du remplissage qui a déstabilisé le parement. Un renfort a donc pu être nécessaire, élevé sous la forme d'un double parement plaqué contre cette paroi orientale, de $5 \mathrm{~m}$ d'épaisseur et entièrement en pierre.

Si le parement en mottes de gazon s'était partiellement effondré avant sa réfection, il n'en reste aucune trace sous le double parement de façade. On peut soumettre l'hypothèse que, malgré un (ou plusieurs renflements) dans la structure de façade, les populations n'ont pas attendu qu'elle s'effondre avant de la renforcer. Par contre, le monoxyle fiché dans le trou de poteau a certainement dû être retiré en préalable au prolongement de la façade, comme en témoigne le remplissage d'un vrac de plaquettes calcaires qui atteste du rebouchage rapide et volontaire de ce trou qui aurait pu devenir un point de faiblesse du nouveau parement. Ce parement est réalisé avec des dalles calcaires plus grandes que celles que l'on peut observer dans le remplissage des caissons.

Même si la matière première est identique (calcaire de Creully), les dalles sont nettement plus grandes en moyenne, tout particulièrement celles de première assise de plus d'un mètre de longueur chacune. Leur recherche a dû s'accompagner d'un approfondissement des fossés-carrières, les dalles étant plus grandes car moins cryoturbées au fond. La découverte de céramiques du Néolithique moyen I (Cerny) brisées, au pied du parement de façade, suggère que cette extension a été réalisée peu de temps après la construction initiale, en tout cas dans la même ambiance culturelle.

Bien que les longs tumulus bretons puissent également être élevés en terre (Cassen et al., 200o), c'est avec les monuments britanniques de type Cotswold-Severn tombs (sud-est du pays de Galles) que le monument 29 offre les points de comparaison les plus pertinents. Ainsi, Beckhampton Road, South Street, Giants Hill, Julliberies Grave ou Skendelby (Scarre, 2006) présentent comme points communs la présence d'un tertre très allongé encadré par deux puissants fossés-carrières latéraux. Ces monuments peuvent recouvrir des structures funéraires antérieures (en bois et brûlées) ou simplement ne comporter aucune structure funéraire (South Street et Beckhampton Road), dans une configuration alors très proche du monument 29 (Scarre, 2006). La construction en mottes de gazon de ces monuments britanniques témoigne comme à Fleury de l'emploi d'un matériau local et abondant. Le principal point d'achoppement reste la datation tardive des monuments de type Cotswold-Severn tombs, entre 3800 et 3500 cal BC. Cet écart de 700 ans à un millénaire (malgré quelques dates plus précoces) ne saurait être interprété comme une perduration d'un courant monumental dont il manquerait les jalons intermédiaires.

La préservation d'un tertre fossilisé par un chemin antique a permis de mettre en évidence l'utilisation de la terre crue (associée à l'herbe et son système racinaire) dans le cadre de la construction d'un monument de très grandes dimensions. La fouille de ce tertre a également été l'occasion d'observer l'association de la terre crue (sous forme de tapis de gazon) avec des plaquettes calcaires, la terre constituant des murets déterminant des caissons et donc l'architecture interne du monument, tandis que la pierre apparaît dans un premier temps uniquement comme un élément de remplissage, non visible. Le parement de façade oriental du tertre, à l'origine monté entièrement en tapis de gazon, n'a probablement pas résisté à la poussée des terres du remplissage. La pierre a alors à ce moment-là joué un rôle de remplacement de la terre, offrant de nouvelles possibilités de construction alliant solidité (les monuments mégalithiques qui n'ont pas été utilisés comme carrières sont encore bien préservés dans le Grand-Ouest) et effet visuel intéressant. Ce type de construction devient exclusif avec le passage au Néolithique moyen II, vers 4400-430o cal BC, pour des sépultures collectives/multiples.

La coloration que prend le substrat sous le tertre du monument 29 (grise sous la partie en mottes de gazon, plus blanche sous les plaquettes) rejoint les observations similaires réalisées sur les autres monuments (gris sous cinq monuments, clair sous l'ensemble des autres). Ils permettent de soupçonner la présence d'un tertre en terre et/ou pierre sur l'ensemble de la surface interne des monuments du site. 




6. Orthophotographie d'un des murets de

refend en tapis de gazon,

qui prend appui sur un

caisson interne comblé à

ce niveau-là de tapis de

gazon.

\section{Références bibliographiques}

CASSEN S. et al., 200o, Éléments d'architecture,

Exploration d'un tertre funéraire à Lannec er

Gadouer (Erdeven, Morbihan), Constructions et

reconstructions dans le Néolithique morbihannais,

Propositions pour une lecture symbolique,

Chauvigny, Association des publications

chauvinoises (Mémoire de la Société de recherches

archéologiques de Chauvigny, XIX), $813 \mathrm{p}$.

Flotté D., 2013, Fleury-sur-Orne, Calvados «les Hauts de l'Orne », rapport d'opération, Inrap-SRA BasseNormandie, $271 \mathrm{p}$.

SCARRE C., 2006, « Tertres funéraires mégalithiques et non-mégalithiques du sud de la Grande-Bretagne », in Joussaume R., Laporte L. ET SCARre C. (DIR.), Origine et développement du mégalithisme de louest de l'Europe, colloque international (Bougon, 26-30

octobre 2002), Bougon, Musée des Tumulus de

Bougon, Niort, conseil général des Deux-Sèvres, p. $187-215$. 